ORIGINAL ARTICLE

\title{
Effects of 20 days of bed rest on the viscoelastic properties of tendon structures in lower limb muscles
}

\author{
K Kubo, H Akima, J Ushiyama, I Tabata, H Fukuoka, H Kanehisa, T Fukunaga
}

Br J Sports Med 2004;38:324-330. doi: 10.1136/bjsm.2003.005595

See end of article for authors' affiliations a ther. a

Correspondence to: K Kubo, PhD, Department of Life Science (Sports Sciences), University of Tokyo, Komaba 3-8-1, Meguro-ku, Tokyo 153-8902, Japan; kubo@ idaten.c.u-tokyo.ac.jp

Accepted 15 April 2003

\begin{abstract}
Objectives: The purpose of this study was to investigate the effects of 20 days' bed rest on the viscoelastic properties of human tendon structures in knee extensor and plantar flexor muscles in vivo.

Methods: Eight healthy men (age: $24 \pm 4$ years, height: $172 \pm 9$ m, body mass: $69 \pm 13 \mathrm{~kg}$ ) carried out a $6^{\circ}$ head-down bed rest for 20 days. Before and after bed rest, elongation (L) of the tendon and aponeurosis of vastus lateralis (VL) and medial gastrocnemius muscles (MG) during isometric knee extension and plantar flexion, respectively, were determined using real-time ultrasonic apparatus, while the subjects performed ramp isometric contraction up to the voluntary maximum, followed by ramp relaxation. The relationship between estimated muscle force $(\mathrm{Fm})$ and tendon elongation $(\mathrm{L})$ was fitted to a linear regression, the slope of which was defined as stiffness. The hysteresis was calculated as the ratio of the area within the $\mathrm{Fm}-\mathrm{L}$ loop to the area beneath the load portion of the curve.

Results: $L$ values above $100 \mathrm{~N}$ were significantly greater after bed rest for $V L$, while there were no significant differences in $L$ values between before and after for MG. The stiffness decreased after bed rest for VL $(70.3 \pm 27.4 \vee 50.1 \pm 24.8 \mathrm{~N} / \mathrm{mm}$, before and after bed rest, respectively; $p=0.003)$ and $M G$ $(29.4 \pm 7.5 \vee 25.6 \pm 7.8 \mathrm{~N} / \mathrm{mm}$, before and after bed rest, respectively; $p=0.054)$. In addition, hysteresis increased after bed rest for VL $(16.5 \pm 7.1 \% \vee 28.2 \pm 12.9 \%$, before and after bed rest, respectively; $p=0.017)$, but not for $M G(17.4 \pm 4.4 \% v 17.7 \pm 6.1 \%$, before and after bed rest, respectively; $p=0.925)$. Conclusions: These results suggested that bed rest decreased the stiffness of human tendon structures and increased their hysteresis, and that these changes were found in knee extensors, but not the plantar flexors.
\end{abstract}

A number of studies have documented that the microgravity environment encountered during spaceflight or simulated by using models of weightlessness induces alterations in skeletal muscle function. ${ }^{12}$ In the absence of weight-bearing activity, strength loss is the most evident consequence of atrophy. These alterations are also accompanied by changes in the mechanical properties of muscle and tendon after spaceflight or bed rest. ${ }^{3-6}$ For example, Witzmann et $a l^{6}$ showed that the maximum rate of tension development decreased in rat soleus muscle following a period of hindlimb immobilisation. Duchateau and Hainaut ${ }^{4}$ reported that the times to peak torque and half relaxation increased after immobilisation for a period of 6 weeks in a standard plaster cast. Furthermore, Anderson et al stated that the observed changes in spindle sensitivity after hindlimb suspension could be due to the decrease in stiffness of the muscle-tendon complex in series with the muscle spindle. Therefore, these observed changes following a period of immobilisation may cause changes in tendon properties, because the chief function of tendon structures is to transfer force produced by the contractile component to the joint and/ or bone connected in series.

So far, most knowledge on the effects of immobility on the tendon and ligament, however, has come from animal studies..$^{7-10}$ Noyes $^{8}$ demonstrated that knee joint immobilisation for 8 weeks decreased the maximum failure load of the anterior crucial ligament. With regard to human tendons, Kubo et al ${ }^{5}$ reported that stiffness of human tendon structures in knee extensors decreased significantly after 20 days of bed rest. However, few studies have investigated the effects of immobilisation on the hysteresis, that is the viscosity, of tendon structures, as far as we know. Furthermore, some researchers have demonstrated that muscle loss after bed rest and spaceflight is greater in the plantar flexors than in thigh muscles such as the knee extensors and flexors. ${ }^{11}$
Considering these findings, we hypothesised that the changes in tendon properties would be greater in the plantar flexors than the knee extensors.

The purpose of this study was to investigate the effects of 20 days of bed rest on the viscoelastic properties of the tendon structures of human knee extensors and plantar flexors in vivo.

\section{METHODS \\ Subjects}

The subjects were eight healthy males (age: $24 \pm 4$ years, height: $172 \pm 9 \mathrm{~m}$, body mass: $69 \pm 13 \mathrm{~kg}$, mean \pm SD), none of whom exercised on a regular basis. Protocols used in this study were approved by the Ethics Committee of the Faculty of Medicine at the University of Tokyo. Prior to the experiment, the details and possible risks of the protocols were explained to the subjects and written informed consent was obtained from each of them.

\section{Bed rest procedures}

The bed rest procedures used in this study have been reported elsewhere. ${ }^{15}$ Briefly, subjects remained in a $6^{\circ}$ head-down tilt bed rest at all times throughout the bed rest period, and did not assume any weight-bearing posture or undertake any physical activities. During transportation the subjects lay on a stretcher. The room temperature of the wards did not exceed $25^{\circ} \mathrm{C}$. All tests were conducted before re-ambulation.

\section{Measurement of viscoelastic properties of tendon structures}

The viscoelastic properties of tendon structures were determined 2 days before the start of bed rest and 1 day after the

Abbreviations: $\mathrm{Fm}$, muscle force; $L$, tendon elongation; $M G$, medial gastrocnemius muscles; VL, vastus lateralis muscles 
end of bed rest. A dynamometer (Myoret, Asics, Japan) was used to determine torque output during isometric knee extension and plantar flexion. For both the actions, subject was instructed to gradually exert increasing force from relaxed to maximal voluntary contraction (MVC) within $5 \mathrm{~s}$, followed by gradual relaxation within $5 \mathrm{~s}$. In the case of the knee extension task, subjects sat in an adjustable chair with back support and the hip joint flexed at an angle of $80^{\circ}$ (full extension $=0^{\circ}$ ) to standardise the measurements and localise the action to the appropriate muscle group. During torque measurements, the hips and back were held tightly in the seat using adjustable lap belts. The axis of the knee joint was aligned with the axis of the lever arm of the dynamometer. The right ankle was firmly attached to the lever arm of the dynamometer with a strap and fixed with the knee joint flexed at an angle of $80^{\circ}$ (full extension $=0^{\circ}$ ). During the plantar flexion task, subject lay prone on a test bench and the waist and shoulders were secured by adjustable lap belts and held in position. The right ankle joint was set at $90^{\circ}$ (anatomical position) with the knee joint at full extension and the foot was securely strapped to a foot plate connected to the lever arm of the dynamometer. The order of the conduction of the two experimental conditions was random for each subject. Prior to the test, subject performed a standardised warm-up and submaximal contractions so as to be accustomed to the test procedure. Each knee extension and plantar flexion task was repeated two or three times per subject with at least 3 min between trials. Torque signals were A/D converted at a sampling rate of $1 \mathrm{kHz}$ (MacLab/8, type ML 780, AD Instrument, Japan) and analysed by a personal computer (Power Macintosh 7200/ 120, Apple Computer). The measured values that are shown below are the means of two trials.

An ultrasonic instrument (SSD-2000, Aloka, Tokyo, Japan) with an electronic linear array probe $(7.5 \mathrm{MHz}$ wave frequency with $80 \mathrm{~mm}$ scanning length; UST 5047-5, Aloka) was used to obtain longitudinal ultrasonic images of vastus lateralis (VL) and medial gastrocnemius (MG) muscles in the right leg using the procedures described by Kubo et al. ${ }^{12}$ The ultrasonographic width and depth resolutions with this probe are 0.67 and $0.4 \mathrm{~mm}$, respectively. The probe was longitudinally attached to the dermal surface of the subject with adhesive tape, which prevented the probe from sliding. ${ }^{13}$ Two sites were selected for measurements: mid-way $(50 \%)$ between the greater trochanter and the lateral epicondyle of the femur for VL and at $30 \%$ of the distance between the popliteal crease and the centre of the lateral malleolus for MG. At these levels, the widths of VL and MG were visualised using the ultrasound apparatus, and the position of one-half of the width was marked with a pen. Longitudinal images were obtained at this position. The ultrasonic images were recorded on videotape at $30 \mathrm{~Hz}$, synchronised with recordings of a clock timer for subsequent analyses. The tester visually confirmed the echoes from the aponeurosis and fascicles. The point at which one fascicle was attached to the aponeurosis $(\mathrm{P})$ was visualised on the ultrasonic images. The $\mathrm{P}$ moved proximally during isometric torque development up to a maximum (fig 1). The displacement of $\mathrm{P}(\mathrm{L})$ indicates the lengthening of the deep aponeurosis and the distal tendon. ${ }^{13}{ }^{14}$

The measured torque (TQ) during isometric knee extension and plantar flexion was converted to force unit (Fm) by the following equation:

$$
\mathrm{Fm}=\mathrm{k} \times \mathrm{TQ} \times \mathrm{MA}^{-1}
$$

where $\mathrm{k}$ is the relative contribution of VL of the knee extensor muscles and MG of the plantar flexor muscles in terms of the ratio of the muscle volume, and MA is the moment arm

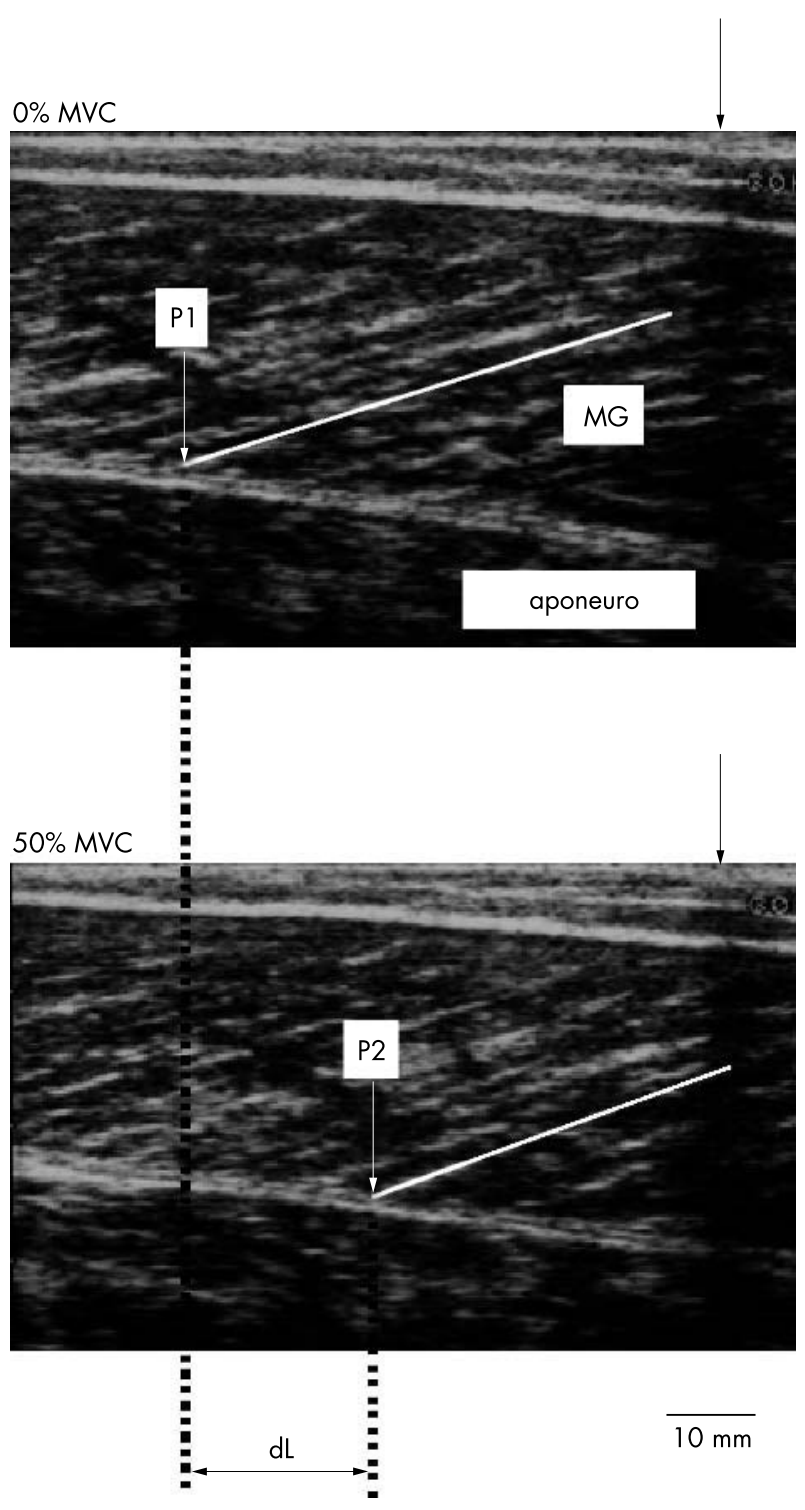

Figure 1 Ultrasonic images of longitudinal sections of the medial gastrocnemius muscle during isometric contraction. The black arrows point to the shadow generated by an echo-absorptive marker attached with adhesive to the skin. The cross-point $(P)$ was determined from the echoes of the deep aponeurosis and fascicles. The point $\mathrm{P}$ moved proximally during isometric torque development from rest $(\mathrm{P} 1)$ to $50 \%$ MVC (P2). The distance travelled by $P(L)$ was defined as the length change of the tendon and aponeurosis during contraction.

length in each of the quadriceps femoris muscles at $80^{\circ}$ and triceps surae muscle at $90^{\circ}$, which was estimated from the limb length of each subject as described by Visser $e t a l^{15}$ and Greive et al. ${ }^{16}$

Fig 2 presents a typical example of the relationship between Fm and L in MG. The Fm-L relationship in the tendon structure was curvilinear consisting of an initial region (toe-region) characterised by a large increase in L with increasing force and a linear region immediately after the toe-region. In the present study, the Fm and L values above $50 \%$ of MVC were fitted to a linear regression equation, the slope of which was adopted as stiffness. ${ }^{13}{ }^{14}$ The Fm-L curves during the ascending and descending phases of force development produced a loop. In the present study, the area of each of the curves under both the ascending and descending phases was calculated. Then, the ratio of the area within the Fm-L loop (elastic energy dissipated) to the 
area beneath the curve during the ascending phase (elastic energy input) was calculated as an index of hysteresis. ${ }^{14}$ Comparison of stiffness and hysteresis values between the two measurements for all subjects revealed no significant difference, with an interclass correlation of $\mathrm{r}=0.88$ and $r=0.86$, and a coefficient of variation of $5.4 \%$ and $9.8 \%$.

\section{Magnetic resonance imaging}

Magnetic resonance imaging was used to measure muscle and tendon cross-sectional areas (AIRIS II, HITACHI Medical, Tokyo, Japan) 2 days before the start of bed rest and 1 day after the end of bed rest. Pre measurement was performed 3 days before bed rest, and post measurement $24 \mathrm{~h}$ after the end of bed rest. All images were taken after 1530 min rest to avoid fluid shifts that might induce interstitial and/or intracellular volume changes. Tl-weighted spin-echo, axial-plane imaging was performed with the following parameters: TR $850 \mathrm{~ms}$, TE $25 \mathrm{~ms}$, matrix $256 \times 256$, field of view $250 \mathrm{~mm}$, slice thickness $10 \mathrm{~mm}$, and interslice gap $0 \mathrm{~mm}$. The subjects were imaged in a supine position with the knee and ankle kept at $0^{\circ}$ (full extension) and $90^{\circ}$ (anatomical position), respectively. Coronal plane images were taken to identify the spina illiaca anterior superior which is the origin of the sartorius. Consecutive axial images were obtained from spina illiaca anterior superior to extremitas distal of tibia. The muscles investigated were as follows: the knee extensors: $m$. rectus femoris (RF), VL, m. vastus intermedius (VI), and $\mathrm{m}$. vastus medialis (VM); the plantar flexors of the ankle: MG, m. lateral gastrocnemius (LG), and $\mathrm{m}$. soleus (SOL). From the series of axial images, outlines of each muscle were traced, and the traced images were transferred to a Macintosh computer (Power Macintosh $7200 / 120$, Apple Computer) for calculation of the anatomical cross-sectional area using a public domain National Institute of Health (NIH) image software package. The muscle volume was determined by summing the cross-sectional area of each image times the thickness $(10 \mathrm{~mm})$. In addition, the tendon cross-sectional area was measured at two positions (one above the patella and the other at $10 \mathrm{~mm}$ proximal from the patella for the quadriceps femoris tendon, and one above the calcareus and the other at $10 \mathrm{~mm}$ proximal from the calcareous for the Achilles tendon). The average of

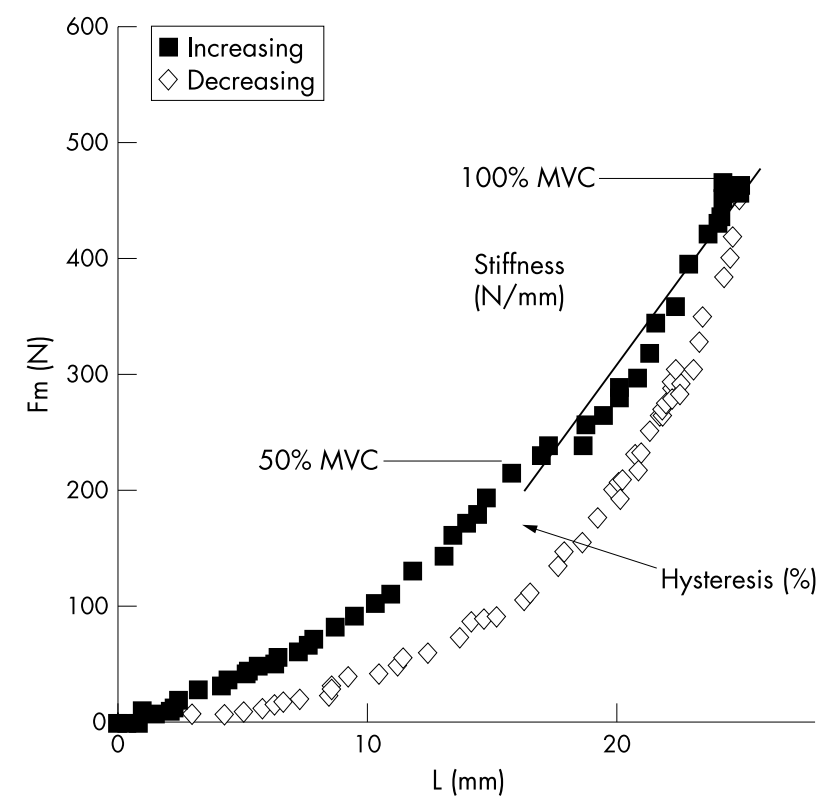

Figure 2 Typical example of estimated muscle force ( $\mathrm{Fm})$-tendon elongation (L) relationship in medial gastrocnemius muscle. cross-sectional areas at the two positions was calculated as representative of tendon cross-sectional area.

Repeatability for the muscle volume and tendon crosssectional area measurements was investigated on 2 separate days in a preliminary study with six young males. There were no significant differences between the test and retest values of the muscle volume or the tendon cross-sectional area. The test-retest correlation coefficient ( $\mathrm{r}$ ) was 0.92 for the muscle volume and 0.97 for the tendon cross-sectional area. The coefficients of variance were $2.4 \%$ for the muscle volume and $1.5 \%$ for the tendon cross-sectional area.

\section{Electromyogram}

The electromyographic activity (EMG) was recorded during the ramp isometric contraction (that is, measurement of tendon properties). Bipolar surface electrodes $(5 \mathrm{~mm}$ in diameter) were placed over the bellies of VL, VM, RF, and biceps femoris muscle (BF) for knee extension and MG, LG, SOL, and tibial anterior (TA) for plantar flexion with a constant interelectrode distance of $25 \mathrm{~mm}$. The positions of the electrodes were marked on the skin by small ink dots. These stained dots ensured the same electrode positioning in each test during the experimental period. The EMG signals were transmitted to a computer (Power Macintosh 7200/120, Apple Computer) at a sampling rate of $1 \mathrm{kHz}$. The EMG was full-wave rectified and integrated for the duration of the contraction to give integrated EMG (iEMG).

\section{Statistics}

Descriptive data included means $\pm S D$. The significance of difference between before and after bed rest was analysed by Student's $t$ test. One-way analysis of variance (ANOVA) was used for the comparison between two measured sites. If the F statistic of the analysis of variance was significant, differences between the two conditions were assessed by a Scheffe test. The level of significance was set at $\mathrm{p}<0.05$.

\section{RESULTS}

Fig 3 shows the relative changes in the muscle volumes of knee extensors and plantar flexors before and after bed rest. The muscle volumes of knee extensors and plantar flexors were significantly reduced by $-10.4 \pm 3.4 \% \quad(p<0.001)$ and $-11.7 \pm 7.0 \% \quad(p=0.004)$, respectively. Furthermore, there were no significant differences in the degree of muscle loss among the constituents of knee extensors and plantar flexors. In addition, no significant changes in the crosssectional areas of quadriceps femoris or Achilles tendons were found after bed rest (table 1).

Table 2 shows the iEMG values of the measured muscles during measurement of the elongation of tendon structures (that is, ramp isometric contractions) before and after bed rest. The EMG activities of each knee extensor and plantar flexor did not differ significantly before or after bed rest. In addition, the iEMG of BF and TA were very small and did not change significantly after bed rest.

Fig 4 shows the relationships between Fm and L before and after bed rest. MVC decreased significantly after bed rest for knee extension $(-20.4 \%)$ and plantar flexion $(-20.6 \%)$ (table 1 ). The $\mathrm{L}$ values above $100 \mathrm{~N}$ were significantly greater after bed rest for VL, while there were no significant differences in $\mathrm{L}$ values between before and after for MG. The stiffness decreased after bed rest for VL $(p=0.003)$ and MG $(p=0.054)$ (table 1$)$. The relative decline in stiffness tended to be greater for VL $(-28.6 \%)$ than for MG $(-13.7 \%)$ $(\mathrm{p}=0.103)$.

Fig 5 shows the relationships between \%MVC and L before and after bed rest. For VL, the \%MVC-L loop was significantly larger after bed rest, although the \%MVC-L loop for MG was not different after bed rest. The hysteresis increased 

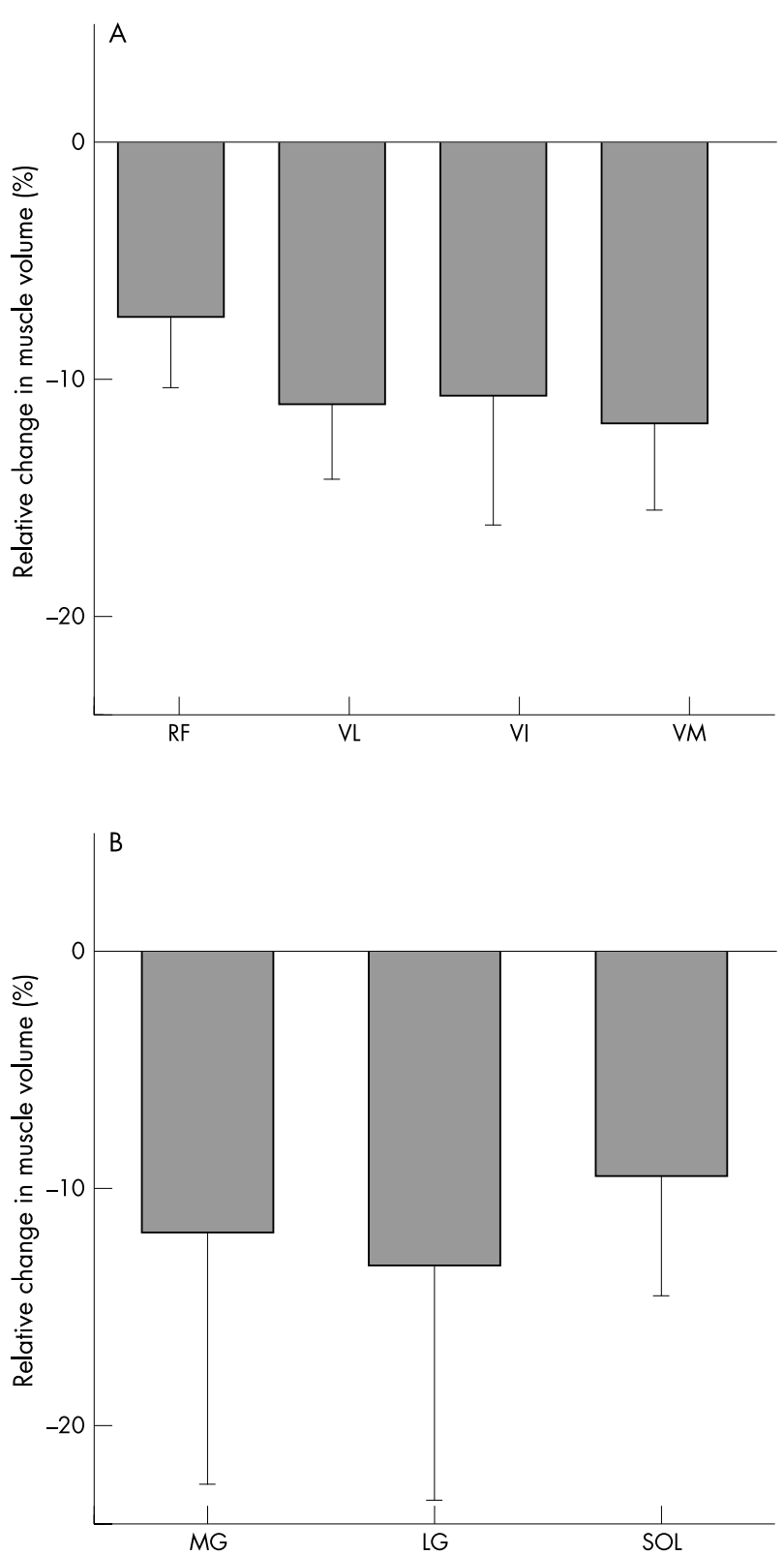

Figure 3 Relative changes in muscle volumes of individual muscles of knee extensors and plantar flexors. All muscle volumes decreased significantly. However, there were no differences in the degree of muscle loss among knee extensors and plantar flexors. LG: m. lateral gastrocnemius; MG: $m$. lateral gastrocnemius; RF: $m$. rectus femoris, SOL: $\mathrm{m}$. soleus; Vl: $\mathrm{m}$. vastus intermedius, VL: $\mathrm{m}$. vastus lateralis, VM: $\mathrm{m}$. vastus medialis.

significantly after bed rest for VL $(p=0.017)$, but not for MG $(p=0.925)$ (table 1$)$. The relative increment of hysteresis was significantly greater for VL $(+93.3 \%)$ than for MG $(+10.4 \%)$ $(\mathrm{p}=0.024)$.

\section{DISCUSSION}

Before interpreting the results obtained, however, we must note the limitations and assumptions of the methodology followed. To calculate the muscle force, we used the ratio of VL to the quadriceps femoris and MG to the plantar flexors in terms of muscle volume as the contribution of the muscle to force development. In the present study, we aimed to investigate the influence of bed rest on the tendon properties rather than force production. In addition, it is noted that there were no significant differences between before and after training in the relative decrease in the muscle volume and electromyograms in each constituent of the quadriceps femoris and plantar flexors (fig 3 and table 2). Furthermore, we also confirmed that little co-contraction occurred either of knee flexors during knee extension or of dorsi-flexors during plantar flexion. This implies that the relative contribution of each constituent to force production was similar before and after bed rest. Therefore, we considered that the muscle force calculation based on the above mentioned assumptions was valid to study the changes of the tendon properties after bed rest.

The Fm-L relationship should be converted to a stressstrain relationship to determine accurately the effect of bed rest on the tendon structures. In this study, no significant changes in the tendon cross-sectional area were found after bed rest (table 1). Most previous studies using animals have documented that few changes in the size of tendons are induced by immobilisation ${ }^{7}{ }^{17}$ or training. ${ }^{18-20}$ With regard to the human tendon, Kubo et $a^{21}$ observed that the quadriceps femoris tendon cross-sectional area did not change after 12 weeks of isometric knee extension training. Therefore, it is unlikely that only 3 weeks of bed rest induced changes in the masses and/or collagen concentrations of the quadriceps femoris and Achilles tendons. The mechanisms causing the decrease in the stiffness remain uncertain, although the bed rest did not induce a significant atrophic change in these tendons. The decreased stiffness might be caused by the bed rest inducing changes in the internal structures of tendons and/or aponeurosis. Rollhauser ${ }^{18}$ pointed out that, after 42 day training for pigs, the only way mature tendons could positively respond to the chronic exercise was to improve the internal structures of tendons. Further, the variability of the mechanical quality of collagen originates from differences in either the cross-link pattern of the collagen or the structure and packing of the collagen fibres. ${ }^{22}$ Considering these findings, this justifies further discussion of the changes in the viscoelastic properties of the tendon structures after bed rest using the Fm-L relation instead of the stress-strain relation.

The average stiffness values were $70 \mathrm{~N} / \mathrm{mm}$ for VL and $28 \mathrm{~N} / \mathrm{mm}$ for MG. On the basis of the above mentioned assumption, we tried to calculate the "normalised stiffness", that is, Young's modulus, using the procedure described by Kubo et al. ${ }^{13}$ The obtained Young's modulus values, $270 \mathrm{MPa}$ for VL and $280 \mathrm{MPa}$ for MG, agreed with our recent observations $^{13}{ }^{14}$ but were considerably lower than those previously reported in vitro. ${ }^{6}{ }^{10}$ This discrepancy can be attributed to the fact that the properties determined in the

Table 1 Measured variables before and after bed rest (mean \pm SD)

\begin{tabular}{|c|c|c|c|c|c|}
\hline & $\operatorname{MVC}(\mathrm{Nm})$ & $L_{\max }(\mathrm{mm})$ & Stiffness $(\mathrm{N} / \mathrm{mm})$ & Hysteresis (\%) & Tendon CSA $\left(\mathrm{mm}^{2}\right)$ \\
\hline \multicolumn{6}{|l|}{ VL } \\
\hline Before & $239 \pm 84$ & $32.8 \pm 5.6$ & $70.3 \pm 27.4$ & $16.5 \pm 7.1$ & $212 \pm 16$ \\
\hline After & $185 \pm 57^{*}$ & $38.3 \pm 4.6^{*}$ & $50.1 \pm 24.8^{*}$ & $28.2 \pm 12.9^{*}$ & $210 \pm 18$ \\
\hline \multicolumn{6}{|l|}{ MG } \\
\hline Before & $114 \pm 18$ & $21.4 \pm 2.9$ & $29.4 \pm 7.5$ & $17.4 \pm 4.4$ & $61.2 \pm 9.7$ \\
\hline After & $92 \pm 29^{*}$ & $22.3 \pm 4.2$ & $25.6 \pm 7.8$ & $17.7 \pm 6.1$ & $59.9 \pm 8.8$ \\
\hline
\end{tabular}


Table 2 The iEMG values $(\mathrm{mVs}$ ) during ramp isometric contraction (mean $\pm \mathrm{SD}$ )

\begin{tabular}{lllll}
\hline & RF & VL & VM & BF \\
\hline $\begin{array}{llll}\text { Thigh } \\
\text { Before }\end{array}$ & $1.23 \pm 0.41$ & $1.44 \pm 0.39$ & $1.41 \pm 0.42$ & $0.11 \pm 0.03$ \\
After & $1.16 \pm 0.54$ & $1.38 \pm 0.31$ & $1.40 \pm 0.44$ & $0.12 \pm 0.03$ \\
\hline & MG & LG & SOL & TA \\
\hline $\begin{array}{llll}\text { Lower leg } \\
\text { Before }\end{array}$ & $1.35 \pm 0.40$ & $1.55 \pm 0.31$ & $1.49 \pm 0.34$ & $0.11 \pm 0.02$ \\
Affer & $1.45 \pm 0.46$ & $1.57 \pm 0.27$ & $1.53 \pm 0.40$ & $0.10 \pm 0.03$ \\
\hline & & & & \\
\hline
\end{tabular}
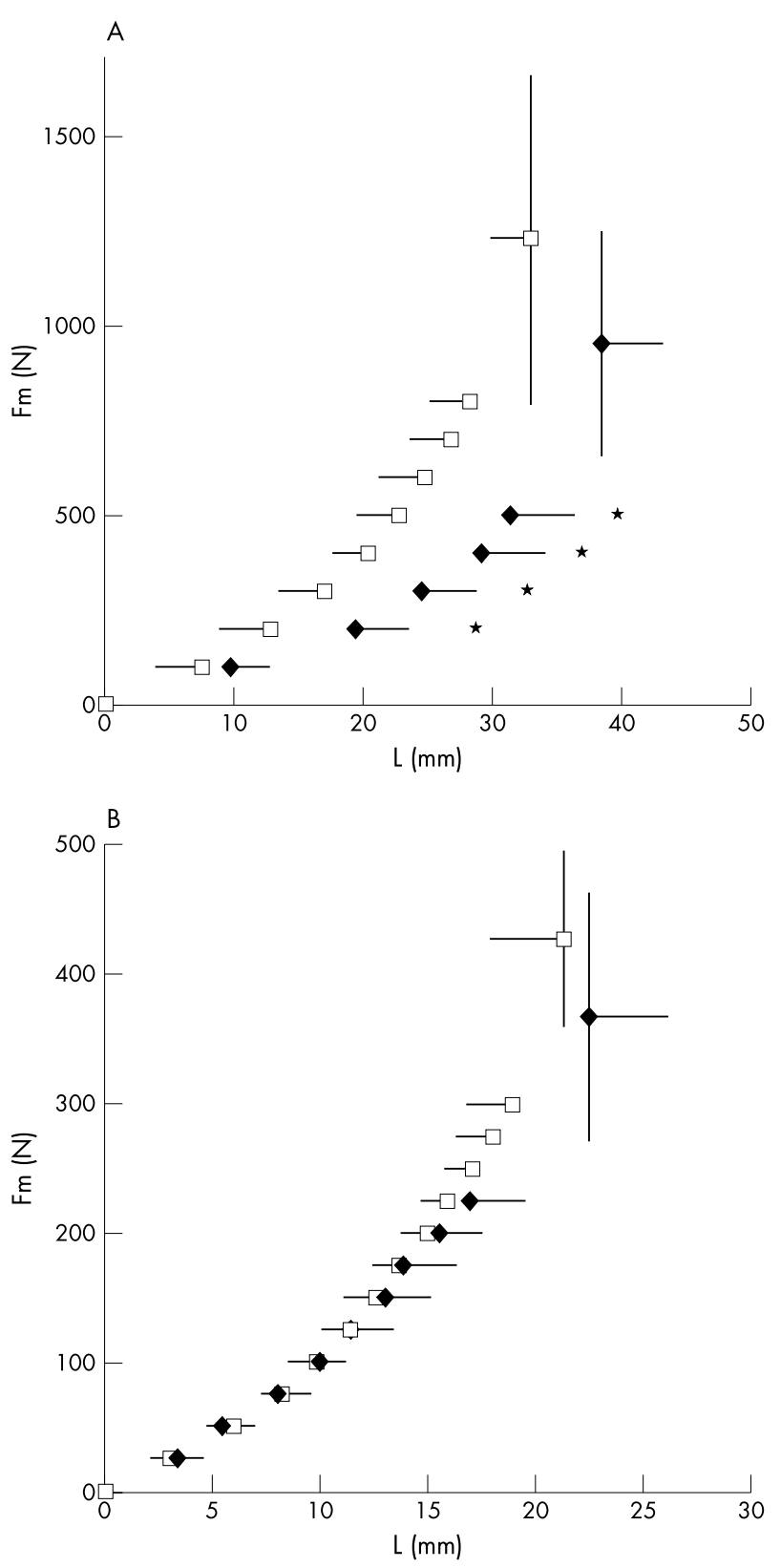

Figure 4 The relationship between $\mathrm{Fm}$ and $\mathrm{L}$ before and after bed rest. For VL (A), the elongations of tendon structures after bed rest (closed symbols) were significantly increased compared to before (open symbols). For MG (B), however, there were no significant differences in the elongations of tendon structures between before and after bed rest. *Significantly greater than before at $p<0.05$. present study represent the elasticity of both outer-tendon and "aponeurosis", while the above quoted research on Young's modulus investigated the outer-tendon only. ${ }^{6}{ }^{10}$ In the present study, the loading and unloading curves during a cyclic force development test produced a loop (that is, hysteresis) as observed in vitro. The area surrounded by the loop represents the energy loss as heat due to internal damping, while the area under the unloading curve is the energy recovered in elastic recoil. The hysteresis determined in the present study averaged $16-17 \%$. This ranks high within the range of $3-20 \%$ reported for 18 species of adult quadrupedal mammals. ${ }^{23}$

Our findings indicated that the 20 days' bed rest led to significant decreases in stiffness of tendon structures in knee extensors $(-28 \%)$, and agreed with previous observations in animals ${ }^{77-20}$ and humans. ${ }^{5}$ Noyes $^{8}$ demonstrated that knee joint immobilisation for 8 weeks decreased the maximum failure load of the anterior crucial ligament. More recently, we reported that the stiffness of human tendon structures in knee extensors decreased significantly after 20 days of bed rest $(-32 \%) .^{5}$ These changes in tendon structures after bed rest might be due to changes in the rate of torque development and the time of peak force. In fact, Bamman et $\mathrm{al}^{24}$ showed a significant decrease of rate of torque development after 14 days of bed rest. Similarly, Duchateau ${ }^{25}$ reported that the maximal rate of tension development was reduced by $31 \%$ in electrically induced and $24 \%$ in voluntary contractions after 5 weeks of bed rest. Therefore, bed rest for 20 days would affect the transmission from muscle fibres to bone, and the ability to produce dynamic force.

In any human isometric tests, the joint angle has been assumed to be constant without direct monitoring of the joint angle. Thus, since isometric contraction of muscle about a joint will produce more or less angular joint rotation in the direction of the intended movement, the resulting tendon and aponeurosis displacement is the result of displacement attributed to both joint angular rotation and contractile tensile loading. Magnusson et $a l^{26}$ demonstrated the importance of accounting for even small amounts of joint motion: despite a rigid frame that was adjusted separately for each subject the average plantar flexion motion was $3.6^{\circ}$. However, it seems reasonable to suppose that there was no difference in this kind of error between before and after bed rest measurements. Furthermore, we aimed to investigate the influence of bed rest on the tendon properties rather than the accurate force production level. In the present study, therefore, we may say that a little overestimation by displacement in knee and ankle joints would have little effect on the present result.

Another interesting finding of this study was that the hysteresis of tendon structures in the knee extensors increased significantly after bed rest. Inversely, our recent observations showed that acute and chronic static stretching decreased the hysteresis of tendon structures. ${ }^{14} 27$ Hysteresis is considered to be an indication of the viscosity of the 

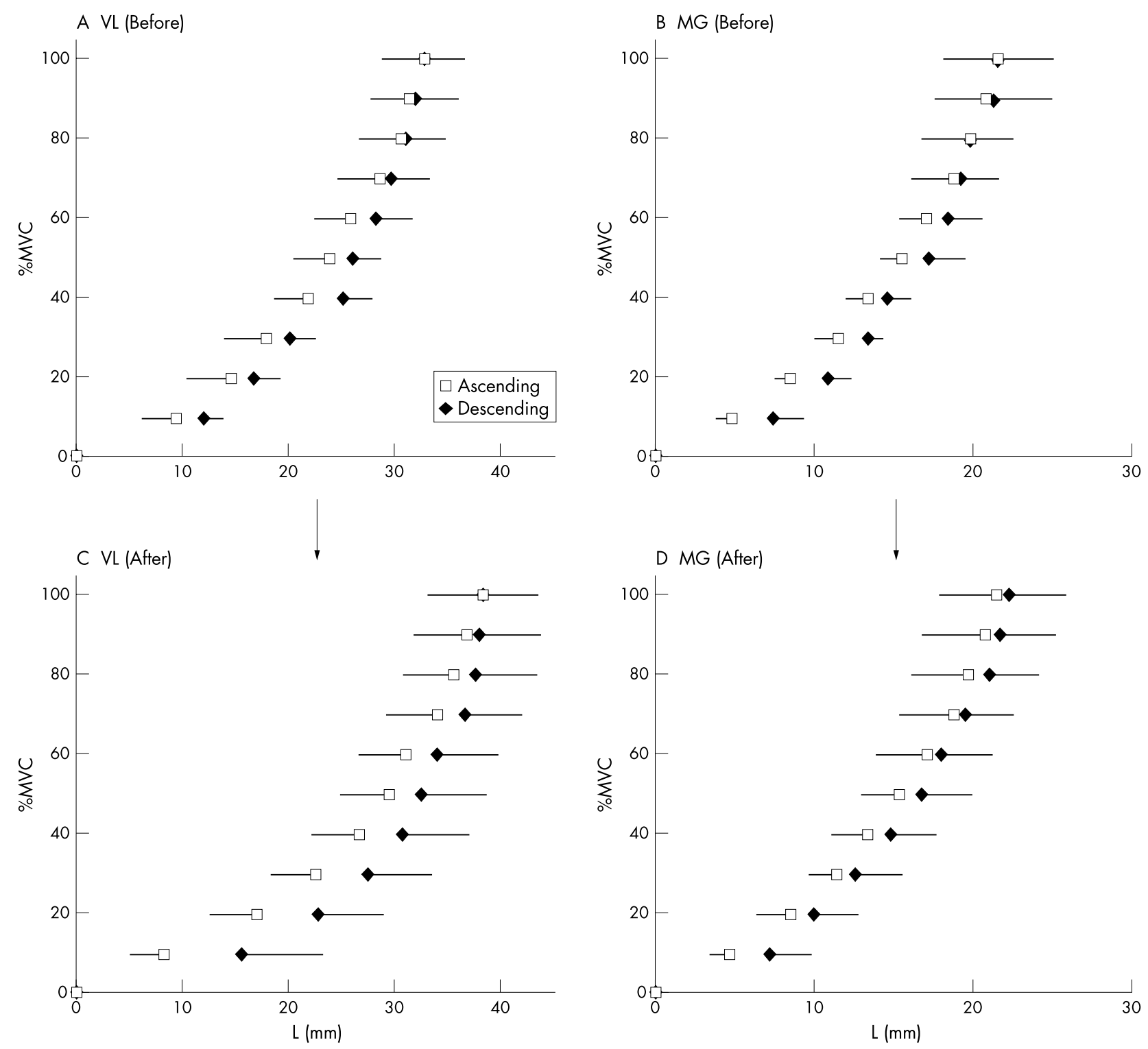

Figure 5 The \%MVC-L curves during ascending and descending phases produced a loop (hysteresis). The hysteresis increased significantly after bed rest for VL, but not for MG. A; vastus lateralis muscle (VL) before bed rest, B; medial gastrocnemius muscle (MG) before bed rest, C; VL affer bed rest, D; MG after bed rest.

tissue. $^{28}$ Therefore, the observed larger hysteresis after bed rest can be interpreted as an increment of viscosity within tendon structures. The mechanisms which resulted in the increase of hysteresis after bed rest are unknown, though in the present study, a change in the structure of the tendons might be involved. Unfortunately, we have no definite information on the changes in the structure of the collagen fibres within the tendons. The hysteresis, that is the area within the loop, represents the energy lost as heat due to internal damping, while the area under the unloaded curve is the energy recovered in the elastic recoil. For example, the value of $28 \%$ obtained in our measurements (after bed rest) would be representative of the percentage energy dissipated as heat in loading-unloading cycles of the tendon during everyday activity. These led us to speculate that bed rest may be an effective way to decrease reused energy during exercise involving a stretch-shortening cycle, by increasing the hysteresis.

On the other hand, the decrease in stiffness after bed rest for 3 weeks tended to be lower in plantar flexors $(-14 \%)$ than in knee extensors $(-28 \%)(p=0.103)$. Further, a significant increase in hysteresis after bed rest was found in knee extensors (93\%), but not in plantar flexors (10\%). Although the reasons for these discrepancies are unknown, there is one possible explanation: they may be due to differences in the plasticity of the various muscles. Some previous studies demonstrated that increases in muscle strength and muscle volume following resistance training were lower in the plantar flexors than in the knee extensors. ${ }^{29}{ }^{30}$ For example, Weiss et a $l^{30}$ showed that 8 weeks of heavy resistance training involving the triceps surae muscles caused an increase of about 13\% in isotonic muscle strength without any concurrent increase in muscularity. Recently, we reported that the elasticity of tendon structures in knee extensors, but not in plantar flexors, was greater in sprinters than in untrained men. ${ }^{12}$ In addition, our recent observations have demonstrated that the increase in stiffness of tendon structures in the plantar flexors was less than in knee extensors. ${ }^{2129}$ Therefore, these discrepancies may be due to different levels of daily activity between knee extensors 


\section{Take home message}

Immobilisation made tendon structures less stiff and more viscous. Furthermore, these changes are greater in the knee extensors than the plantar flexors.

and plantar flexors. Regardless, further investigations are needed to clarify this point.

In conclusion, these results suggest that bed rest decreased the stiffness of human tendon structures and increased their hysteresis, and that these changes were found in knee extensors, but not in plantar flexors.

\section{Authors' affiliations}

K Kubo, J Ushiyama, H Kanehisa, Department of Life Science,

University of Tokyo, Meguro, Tokyo, Japan

H Akima, Research Center of Health, Physical Fitness and Sports,

Nagoya University, Aichi, Japan

I Tabata, Laboratory of Exercise Physiology, Division of Health Promotion, National Institute of Health and Nutrition, Shinjuku, Tokyo, Japan

H Fukuoka, Department of Health Administration, School of Health Science and Nursing, Faculty of Medicine, University of Tokyo, Bunkyo, Tokyo, Japan

T Fukunaga, Department of Human Sciences, Waseda University, Tokorozawa, Saitama, Japan

This study was funded in part by Ground Research for Space Utilization, promoted by the National Space Development Agency and Japan Space Forum.

Conflict of interest: none declared.

\section{REFERENCES}

Akima $\mathrm{H}$, Kubo $\mathrm{K}$, Kanehisa $\mathrm{H}$, et al. Leg-press resistance training during 20 days of $6^{\circ}$ head-down-tilt bed rest prevents muscle deconditioning. Eur J Appl Physiol 2000;82:30-8

2 LeBlanc A, Schonfeld E, Evans HJ, et al. Regional changes in muscle mass following 17 weeks of bed rest. J Appl Physiol 1992:73:2172-8.

3 Anderson J, Almeida-Silveira MI, Perot C. Reflex and muscle adaptations in rat soleus muscle after hindlimb suspension. J Exp Biol 1999;202:2701-7.

4 Duchateau J, Hainaut K. Electrical and mechanical changes in immobilized human muscle. J Appl Physiol 1987;62:2168-73.

5 Kubo K, Akima H, Kouzaki $M$, et al. Changes in the elastic properties of tendon structures following 20 days bed-rest in humans. Eur J Appl Physiol 2000;83:463-8

6 Witzmann FA, Kim DH, Fitts RH. Hindlimb immobilization: length-tension and contractile properties of skeletal muscle. J Appl Physiol 1982:53:335-45.

7 Almeida-Silveira MI, Lambertz D, Perot C, et al. Changed in stiffness induced by hindlimb suspension in rat Achilles tendon. Eur J Appl Physiol 2000;81:252-7.
8 Noyes FR. Functional properties of knee ligaments and alterations induced by immobilization. Clin Orthop Rel Res 1977;123:210-42

9 Tipton CM, Vailas AC, Matthes RD. Experimental studies on the influences of physical activity on ligaments, tendons and joints: a brief review. Acta Med Scand Suppl 1986;711:157-68.

10 Woo SLY, Harwood FL, Akeson WH. The effect of immobilization on collagen turnover in connective tissue: a biochemical-biomechanical correlation. Acta Orthop Scand 1982;53:325-32.

11 LeBlanc A, Rowe R, Schneider V, et al. Regional muscles loss after short duration spaceflight. Aviat Space Environ Med 1995;66:1151-4.

12 Kubo K, Kanehisa H, Kawakami Y, et al. Elasticity of tendon structures of lower limbs in sprinters. Acta Physiol Scand 2000;168:327-35.

13 Kubo K, Kawakami Y, Fukunaga T. The influence of elastic properties of tendon structures on jump performance in humans. J Appl Physiol 1999;87:2090-6.

14 Kubo K, Kanehisa H, Kawakami Y, et al. Influence of static stretching on viscoelastic properties of human tendon structures in vivo. J Appl Physiol $2001 ; 90: 511-9$.

15 Visser JJ, Hoogkamer JE, Bobbert MF, et al. Length and moment arm of human leg muscles as a function of knee and hip-joint angles. Eur J Appl Physiol 1990;61:453-60.

16 Greive DW, Pheasant S, Cavagna PR. Prediction of gastrocnemius length from knee and ankle joint posture. In: Asmussen, Jorgensen K, eds. Biomechanics VI-A 1978:405-12.

17 Amiel D, Woo SLY, Harwood FL, et al. The effect of immobilization on collagen turnover in connective tissue: a biochemical-biomechanical correlation. Acta Orthop Scand 1982;53:325-32.

18 Rollhauser H. Funktionelle Anpassung der Sehnenfaser im submikroskopischen Bereich. Z Anat Anzeig 1954;51:318-22.

19 Viidik A. Simultaneous mechanical and light microscopic studies of collagen fibers. Z Anat Entwick 1972;136:204-12.

20 Woo SL, Gomez MA, Amiel D, et al. The effects of exercise on the biomechanical and biochemical properties of swine digital flexor tendons. J Biomech Eng 1981;103:51-6.

21 Kubo K, Kanehisa H, Fukunaga T. Effects of different duration isometric contractions on tendon elasticity in human quadriceps muscles. J Physiol 2001;536:649-55

22 Danielsen CC, Andereassen TT. Mechanical properties of rat tail tendon in relation to proximal-distal sampling position and age. J Biomech 1988;21:207-12.

23 Pollock CM, Shadwick RE. Relationship between body mass and biomechanical properties of limb tendons in adult mammals. J Appl Physiol 1994;35:1016-21.

24 Bamman MM, Clarke MS, Feeback DL, et al. Impact of resistance exercise during bed rest on skeletal muscle sarcopenia and myosin isofor distribution. J Appl Physiol 1998;84:157-63.

25 Duchateau J. Bed rest induces neural and contractile adaptations in triceps surae. Med Sci Sports Exer 1995;27:1581-9.

26 Magnusson SP, Aagaard P, Rosager S, et al. Load-displacement properties of the human triceps surae aponeurosis in vivo. J Physiol 2001;531:277-88.

27 Kubo K, Kanehisa H, Fukunaga T. Effect of stretching training on the viscoelastic properties of human tendon structures in vivo. J Appl Physiol 2002;92:595-601

28 Butler DL, Grood ES, Noyes FK, et al. Biomechanics of ligaments and tendons. Exer Sports Sci Rev 1978;6:125-81.

29 Kubo K, Kanehisa H, Fukunaga T. Effects of resistance and stretching training programs on the viscoelastic properties of tendon structures in vivo. J Physiol 2002;538:219-26.

30 Weiss LW, Clark FC, Howard DG. Effects of heavy-resistance triceps surae muscle training on strength and muscularity of men and women. Phys Ther 1996;68:208-13. 